\title{
Questes
}

vestes Revue pluridisciplinaire d'études médiévales

\section{L'autorité des hommes âgés dans les exempla}

\section{Anne-Laure Lallouette}

\section{(2) OpenEdition \\ Journals}

\section{Édition électronique}

URL : http://journals.openedition.org/questes/4104

DOI : 10.4000/questes.4104

ISSN : 2109-9472

\section{Éditeur}

Les Amis de Questes

\section{Édition imprimée}

Date de publication : 15 janvier 2006

Pagination : 49-54

ISSN : 2102-7188

\section{Référence électronique}

Anne-Laure Lallouette, "L'autorité des hommes âgés dans les exempla », Questes [En ligne], 8 | 2006,

mis en ligne le 01 janvier 2014, consulté le 21 avril 2019. URL : http://journals.openedition.org/ questes/4104; DOI : 10.4000/questes.4104

Ce document a été généré automatiquement le 21 avril 2019.

(c) Association des amis de «Questes » 


\title{
L'autorité des hommes âgés dans les exempla
}

\author{
Anne-Laure Lallouette
}

Le XIII ${ }^{\mathrm{e}}$ siècle est celui de l'apogée des exempla; ces textes sont indissociables des sermons dans lesquels ils se trouvent insérés, et ne peuvent être compris sans être replacés dans le contexte du renouveau de la prédication de cette époque. Ils sont liés à ce que les historiens ont appelé la "parole nouvelle $»^{1}$, c'est-à-dire cette nouvelle manière de s'adresser à la foule, afin d'être compris le mieux possible par elle, en ayant recours à des thèmes qui lui sont familiers. Un prédicateur de cette époque indique que le caractère prosaïque, ludique ou particulièrement exemplaire d'une anecdote insérée dans un sermon peut avoir pour vertu de réveiller un auditoire qui aurait tendance à s'ennuyer, voire à s'assoupir au cours du prêche. ${ }^{2}$

2 La définition la plus classiquement admise de l'exemplum est donnée dans le tome qui lui est consacré de la Typologie des sources du Moyen Âge occidental: "un récit bref donné comme véridique et destiné à être inséré dans un discours (en général un sermon) pour convaincre un auditoire par une leçon salutaire $»^{3}$. Il constitue un outil précieux pour l'historien, dans la mesure où il donne à voir ce que les clercs pensent être les représentations collectives des fidèles. Il sert de miroir dans lequel apparaît, en partie, la vie quotidienne des paroissiens et apporte de nombreux renseignements sur la place des personnes âgées dans la société.

3 Or, il est intéressant de remarquer que, lorsqu'ils mettent en scène la vieillesse masculine, les prédicateurs octroient une très grande autorité à cette dernière. Quels sont les fondements de cet ascendant des vieillards sur les générations suivantes et comment se traduit-t-il ? En premier lieu, les auteurs accordent une place toute particulière aux hommes âgés dans les textes, qu'ils justifient par la sagesse et le poids de leurs paroles. Ce statut a pour conséquence de faire de ces personnages des êtres écoutés et respectés. 


\section{La parole sacrée du vieil homme}

4 À l'inverse des textes littéraires, les exempla laissent une large place à des personnages âgés positifs et font d'eux des représentants privilégiés de la sagesse et de l'opinion la plus juste. Les hommes d'Église, plus accoutumés à la fréquentation des Écritures saintes ou des ouvrages de la tradition patristique que les auteurs des cours aristocratiques, sont profondément marqués par l'image des patriarches bibliques et l'idée que les Verba Seniorum, les paroles des anciens, sont dignes de foi et de respect. C'est pourquoi, de vénérables vieillards incarnent dans les textes une parole sacrée, véritable et respectée. La bouche du vieillard devient, ponctuellement, le vecteur par lequel le prédicateur fait passer les dogmes et les règles de l'Église.

5 Un des éléments qui traduisent, dans les exempla, ce respect de la parole des aînés est le fait que l'autorité des vieillards se trouve souvent convoquée lorsqu'il s'agit d'attester de faits anciens ou bien lorsque l'on cherche à établir la vérité à propos de faits présents. Ainsi, lorsque Étienne de Bourbon s'interroge à propos de la moralité et du comportement d'un des villageois dont il a la charge, il prend ses renseignements : « de la bouche de quelques anciens du pays, hommes très dignes de foi $»^{4}$. La parole des vieux voisins a force de vérité simplement parce que ceux qui la prononcent ont plus d'expérience et de connaissances. Il est d'ailleurs habituel dans les procédures juridiques de ce temps d'inviter à témoigner en priorité les personnes les plus dignes de confiance, parmi lesquelles les plus vieux figurent en bonne place.

6 Cette vieillesse investie d'autorité tient une place originale dans les textes, dans lesquels elle peut devenir un outil rhétorique. En effet, lorsqu'un auteur choisit de faire dire quelque chose à un homme âgé, c'est, le plus souvent, pour lui faire énoncer quelques dogmes ou pour garantir la véracité du propos. Ceci est particulièrement visible, par exemple, dans la construction de l'œuvre Disciplina clericalis de Pierre Alphonse ${ }^{5}$, qui est un juif converti espagnol de la fin du $\mathrm{xI}^{\mathrm{e}}$ siècle. Cet ouvrage, considéré comme le premier recueil de textes exemplaires, connaît un très grand succès aux siècles suivants et est très fréquemment repris par ses successeurs ${ }^{6}$. Or, toute la structure de l'œuvre repose sur un dialogue entre un père âgé (Senex), dont le propos est toujours investi d'autorité et son fils (Juvenis). Le plus âgé, dans un souci didactique, guide son fils de telle sorte qu'il adopte la conduite la plus juste, c'est-à-dire la plus en adéquation avec ce que l'on peut attendre d'un chrétien. L'expérience liée à son grand âge fait qu'il possède des capacités et des connaissances que les plus jeunes ne peuvent pas avoir, et dont il doit, d'après le clerc, les faire profiter. Le fait de se référer aux vieillards n'est pas un acte anodin pour les prédicateurs : il leur permet de consolider leur discours et d'étayer leur argumentation.

7 Cette conception du grand âge comme le dépositaire de l'autorité morale et intellectuelle est renforcée au XIII ${ }^{\mathrm{e}}$ siècle dans les milieux ecclésiastiques par le regain d'intérêt porté aux Vitae patrum, traduites alors en langue vernaculaire. Cette volumineuse traduction rassemble de nombreux récits et anecdotes ayant trait aux Pères de l'Église, et trouve de très nombreuses correspondances dans les recueils d'exempla. Or, les pères de l'Église sont ceux qui, depuis le concile de Latran de 649, sont également appelés par les ecclésiastiques, les Auctoritates, c'est-à-dire ceux dont l'autorité ne peut pas être contestée. Ces Pères de l'Église sont très souvent de vénérables vieillards, particulièrement ceux cités les Verba seniorum, recueil de citations des Pères du désert, compilées au cours $\mathrm{du} \mathrm{VI}^{\mathrm{e}}$ siècle. Les auteurs de sermons utilisent très fréquemment ces 
textes, citant tour à tour des anecdotes qui y sont rapportées, ou s'en servant comme autorité de référence au moment où ils expliquent l'exemplum à la lumière des textes bibliques et patristiques. Jean Gobi dans la Scala Coeli (L'Échelle du ciel), qu'il compose en Provence entre 1322 et 1330, par exemple, commence fréquemment ces exempla par " Dicitur in Vitas patrum » $\left(\mathrm{n}^{\circ} 12,24,91\right)$ ou « Legitur enim in Vitas patrum » $\left(\mathrm{n}^{\circ} 275\right)^{7}$. Un vieil homme intervient alors systématiquement, comme protagoniste ou bien pour rapporter un récit qui ne met pas nécessairement en scène des personnes âgées, sa présence servant de caution morale au récit.

8 Le poids de la tradition biblique et patristique n'est pas le seul élément qui permette d'expliquer les représentations positives de la sénescence que propose la littérature exemplaire. Si les exempla laissent une place moins modeste que les fabliaux ou les récits courtois à de bons vieillards, c'est aussi parce que les hommes d'Église regardent le dernier âge de la vie comme celui d'une spiritualité plus épanouie, au cours duquel la proximité du trépas invite le fidèle à se tourner plus complètement vers Dieu.

\section{Des guides pour les plus jeunes}

$9 \quad$ La sagesse, depuis les patriarches bibliques, semble devoir s'acquérir avec le nombre des années. La figure du vieux sage prompt à prodiguer ses conseils aux plus jeunes se retrouve dans la plupart des sources religieuses, y compris dans les exempla où un personnage âgé peut avoir à fournir à un jouvenceau la sagesse dont sa jeunesse le prive. Le recueil d'exempla Ci-nous dit signale d'ailleurs que l'ignorance peut être "escuse par defaute d'age », excusée par le peu d'années vécues, et donc par le manque d'expérience ${ }^{8}$. Ce thème est illustré par un exemplum d'Étienne de Bourbon:

«Dans une ville qui servait de résidence à un grand roi, se tenait, dit-on, une foire très importante. Le fils du roi s'en alla avec ses compagnons visiter les étalages, pour voir s'il ne trouverait pas quelques articles à son goût. Comme il traversait le champ de foire, il aperçut une boutique merveilleusement ornée, décorée de draps d'or, et de soie, et cependant elle ne contenait rien. Très étonné, il entra.

À l'intérieur se trouvait seulement un vieillard, assis sur une chaire élevée et tenant à la main un livre, dans lequel il lisait. Le jeune prince lui demanda ce que cela signifiait. Il répondit que cette boutique était celle où se vendaient les marchandises les plus rares et les plus précieuses de toute la foire, surtout pour les hommes appelés à diriger les autres.

« Si vous en voulez, ajouta-t-il, je vous en vendrai pour cent livres et plus.

- Quelles sont donc ces marchandises mystérieuses? fit le prince.

- Ce sont la sagesse et la prudence. »

Le jeune homme lui offrit alors cent livres, et le vieillard écrivit sur une petite cédule ces simples mots : "Dans toutes vos actions, considérez, avant de les faire, à quel résultat elles peuvent vous conduire.» Puis, il lui dit, en lui remettant cette maxime, que, s'il la gardait toujours sous ses yeux, elle lui serait plus avantageuse que la possession d'un royaume.

Bientôt le roi vint à mourir, et son fils lui succéda. Le nouveau monarque fit reproduire le contenu de la cédule tout autour de lui, sur les tables, sur les nappes, sur les serviettes et sur tous les objets à son usage.

Or, il arriva que ses barons ourdissent une conspiration contre lui, et firent un pacte avec son barbier pour que cet homme le tuât. Le barbier voulut tenir sa promesse. Mais, au moment où il rasait le prince et se disposait à lui couper la gorge, les mots inscrits sur la serviette frappèrent ses yeux : il s'arrêta, frémit, et fut saisit d'un tremblement extraordinaire. 
Cela parut suspect au roi, qui le fit emprisonner et soumettre à la question. Contraint de dire la vérité, il dénonça ses complices, qui furent arrêtés à leur tour, et, grâce à cette révélation, il échappa lui-même à la mort.

La prédiction du vieux marchand de sagesse s'était réalisée. ${ }^{9}$

Si l'objectif de cet exemplum est d'exalter la prudence, comme vertu essentielle dans la conduite du chrétien, qui doit peser à tout instant la portée de ses actes, il permet surtout de constater qu'un vieillard peut être présenté comme l'incarnation même de la sagesse. Ici, le grand âge du commerçant, sa position en hauteur - à l'image du prédicateur en chaire -, le terme latin est d'ailleurs " cathedra ", et le livre qu'il consulte sont autant de symboles de sagesse et de maturité intellectuelle, qui servent de pendant à la fougue désordonnée du jeune homme. À cela s'ajoute son don, sinon de divination, au moins de clairvoyance, qui le rend capable d'anticiper l'avenir ou de comprendre le sens caché des événements. Cet aspect du texte le rapproche du fabliau La bourse pleine de sens ${ }^{10}$, dans lequel un vieux marchand, à qui un mari adultère vient acheter la «bourse pleine de sens ", c'est-à-dire de sagesse, que sa femme lui a demandé, comprend que celle-ci voudrait que son époux quitte sa maîtresse. Il donne alors au mari volage des conseils qui vont le conduire à quitter sa maîtresse fort vénale et retrouver la tendresse de son épouse légitime.

Cette prévalence intellectuelle des plus âgés est un des critères fondamentaux qui déterminent leur autorité. Mais cette dernière n'est pas gratuite ou vaine, et ne se trouve justifiée que lorsqu'elle devient effectivement profitable pour autrui, en particulier pour plus jeune que soi.

11 Finalement, lorsque les clercs reprennent à leur compte une opposition entre les différentes générations, qui est un motif récurrent de la littérature de ce temps, ils en détournent les codes et la font tourner à l'avantage des personnes âgées. Jean Gobi, par exemple, choisit d'illustrer avec ironie dans la Scala Coeli, la victoire des aînés sur une jeunesse trop impertinente :

«Un maître versé dans le maniement des armes avait un élève qui se distinguait de tous les autres, celui-ci alors qu'il eut assimilé tous les aspects de cet art et sa pratique, dit à son maître qu'il le surpassait en tout, ce qu'il voulait prouver en faisant organiser un duel. Alors qu'ils étaient tous les deux pris par les préparatifs du combat avec des épées d'entraînement, le maître dit à son élève : "Je te demande si c'est là ta manière de me montrer maintenant ta vraie amitié : dans la mesure où tu es prêt à être le vaincu et me laisser être la vainqueur, alors que tu es jeune et moi vieux, je te prouve que je suis plus agile." Alors le disciple répond : "Ce n'est pas là mon intention, parce que si je te battais, toi qui a de l'expérience, cela me ferait acquérir une gloire perpétuelle et je pourrais accéder au statut de maître. »

Le maître s'étonne de cette attitude et ampute une jambe à l'impudent alors que celui-ci se retourne, en lui disant que c'est une punition pour son manque de loyauté ${ }^{11}$. L'apprenti pâtit d'avoir voulu renverser l'ordre des générations et accaparer une parcelle de l'autorité de son maître.

12 Les exempla, en insistant sur la prédominance de la vieillesse, renversent les images habituelles d'un grand âge assez généralement honni, et semblent pencher plus volontiers en faveur de ceux dont le décès prochain les rend plus proches de Dieu. 


\section{NOTES}

1. Jacques LE Goff et Jean-Claude schmitt, «Au XIII siècle, une "parole nouvelle" ", in Histoire vécue du peuple chrétien, Jean DelumEAu (dir.), Paris, Privat, tome 1, 1979, pp. 257-279.

2. Il s'agit de JACQUES DE VITRY, The "Exempla», or illustrative stories from "sermones vulgares " of Jacques de Vitry, Thomas Frederick CRANE (éd.), London, D. Nutt, 1890.

3. Claude BRÉMOND, Jacques LE GOFF et Jean-Claude SCHMITT, L'« exemplum ", dans Typologie des sources du Moyen Âge occidental, Léopold GÉNICOT (dir.), fasc. 40, Turnhout, Brepols, 1982, pp. 37-38.

4. Traduction d'Albert LECOY DE LA MARCHE (éd.), Le rire du prédicateur, Jacques BERLIOZ (présentation et réédition), Turnhout, Brepols, 1999, p. 83. Texte latin: ÉTIENNE DE BOURBON, Anecdotes historiques, légendes et apologues tirés du recueil inédit d'Étienne de Bourbon, Albert LECOY DE LA MARCHE (éd.), Paris, H. Loones, 1877, 430, p. 374 : « ut audivi ab antiquis fidelibus »

5. PETRUS ALFONSI, The disciplina clericalis, Eberhard HERMES (éd.), Berkeley et Los Angles, University of California Press, 1977.

6. Jacques Berlioz et Marie-Anne polo De BEAULIEU, « La capture du récit. La Disciplina clericalis de Pierre Alphonse dans les recueils d'exempla (XIII ${ }^{\mathrm{e}}-\mathrm{XIV}^{\mathrm{e}}$ s.) », dans C.R.I.S.O.L., Nanterre, n.s., 4, 2000, pp. 33-58.

7. JEAN Gobi, La "Scala coeli" de Jean Gobi, Marie-Anne polo DE BeAulieu (éd.), Paris, Éd. du Centre national de la recherche scientifique, 1991.

8. Ci-nous dit, "Ci nous dit » : recueil d'exemples moraux, 2 tomes, Gérard BLANGEZ (éd.), Paris, Picard, Société des Anciens Textes Français, 1979 et 1987, tome 1, p. 35.

9. Albert LECOY DE LA MARCHE (éd.), Le rire du prédicateur, op. cit., pp. 66-67.

10. Cf. JEAN LE GALOIS, La bourse pleine de sens, in Nouveau recueil complet des fabliaux, N.R.C.F., publié par Willem NOOMEN et Nicolas VAN DEN BOOGAARD, en collaboration avec L. GESCHIERE et H. B. SOL, Assen, Van Gorcum, 1983, 12 volumes, tome 2, pp. 107-143.

11. JEAN GOBI, La "Scala coeli", op. cit., chapitre 73 : « De Amicicia », p. 193.

\section{INDEX}

Keywords : guide, young, speech, sacred, authority, age, sermon, wisdom

Mots-clés : guide, jeune, parole, sacré, autorité, exemplum, âge, sermon, sagesse 